
\title{
28 Research Suare \\ Title High Flow Nasal cannula oxygen therapy in a general pediatric (non-intensive care) setting
}

\author{
Louise Kooiman \\ Isala \\ Roelien Reimink \\ Isala \\ Veerle Langenhorst \\ Isala \\ Paul Brand \\ Isala \\ Jolita Bekhof ( $\sim$ j.bekhof@isala.nl ) \\ Isala
}

Research article

Keywords: Bronchiolitis, Respiratory Syncytial Virus, Pneumonia, Respiratory failure, High flow nasal cannula

Posted Date: November 18th, 2019

DOl: https://doi.org/10.21203/rs.2.17416/v1

License: (c) (i) This work is licensed under a Creative Commons Attribution 4.0 International License.

Read Full License 


\section{Abstract}

Background: High flow nasal cannula therapy (HFNC) is being used increasingly for oxygen delivery in children with impending respiratory failure, however solid evidence of its effectiveness is sparse. Moreover, data on safety regarding its use outside of the Pediatric Intensive Care Unit (PICU), with flowrates exceeding $1 \mathrm{~L} / \mathrm{kg}$ is lacking.

Methods: Retrospective chart review at the pediatric ward of Isala, a general teaching hospital in Zwolle, The Netherlands, $100 \mathrm{~km}$ away from the nearest PICU. All children $<18$ years with impending respiratory failure treated with HFNC between January 2015 and May 2016 were included. A flowrate of 2 $\mathrm{L} / \mathrm{kg} /$ minute for the first $10 \mathrm{~kg}$ was used; with $0.5 \mathrm{~L} / \mathrm{kg}$ for every $\mathrm{kg}>10 \mathrm{~kg}$ and a maximum of $50 \mathrm{~L} / \mathrm{min}$. A pediatric early warning score (PEWS) comprising vital functions and work of breathing (0-28 points) was used to assess severity of respiratory distress. Treatment failure was defined as referral to the PICU.

Results: In the 16-month study period HFNC was used during 41 hospital admissions in 39 patients (64.1\% male), median age 6.3 months (interquartile range, IQR 3-20.6). Median (IQR) PEWS at the start of HFNC was $8.5(7-10)$. Patients were diagnosed with bronchiolitis $(70.7 \%)$, pneumonia $(24.4 \%)$ or asthma (4.9\%). In 18 cases (43.9\%) HFNC failed, with referral to a PICU. No clinical variables (age, comorbidity, PEWS at admission or start of HFNC) nor improvement of the PEWS after 2 hours of HFNC were associated with treatment failure. We found no association between treatment failure and the start of HFNC at an earlier stage or at lower PEWS (odds ratio 1.03; 95\% confidence interval 0.82-1.30; $\mathrm{p}=0.80$ ). There were no safety issues, no cases with air leak or other complications.

Conclusions: This small study suggests that HFNC can be safely used and initiated in a general pediatric department. We were unable to find clinical factors that predicted HFNC success. We recommend not to restrict evaluation of the effect of HFNC in studies to short-term (2 hours), but also after longer duration, at least 24 hours.

\section{Background}

High flow nasal cannula therapy (HFNC) is being used increasingly for oxygen delivery in children and adults with impending respiratory failure (1). Usually, supplemental oxygen is being given as dry gas via low flow nasal prongs (LFNP), with a maximum flow rate of $2-3 \mathrm{~L} / \mathrm{min}$. Higher flow rates are uncomfortable and painful for the patient. With HFNC, heated humidified flow can be given comfortably at higher flow rates, matching or exceeding the patients inspiratory flow rate, thus limiting entrainment of room air (1).

Many pediatric departments have implemented HFNC despite absence of solid evidence of its effectiveness and safety $(2,3)$. Apart from two recently published randomized controlled trials $(4,5)$, most pediatric data on HFNC come from descriptive case series in pediatric intensive care units (PICU) or emergency departments in tertiary settings (6). The few studies from general pediatric wards are observational studies in small groups of children with bronchiolitis in tertiary care hospitals with direct 
access to a PICU in the same hospital. $(6,7)$ Potential risks of HFNC include abdominal distension, infections from the heated humidifying system or pneumothorax $(6,8)$. As these adverse events have been reported in very low frequency, HFNC is regarded as safe treatment (1). However, concerns exist about the setting in which HFNC is being used, and feasibility and safety of its use on general pediatric wards without a nearby PICU is uncertain. One of the concerns is that a delay in initiating invasive ventilation may lead in an increase of mortality and morbidity in cases of respiratory failure, which has been seen in adults (9). In addition, there is debate concerning several aspects of the use of HFNC such as the timing of initiating HFNC. Some restrict the use of HFNC to more severe respiratory distress, while others advocate early use of HFNC, when respiratory distress is not yet severe, possibly preventing later deterioration (7). In the recent randomized controlled trials, HFNC was initiated early, at peripheral capillary oxygen saturation $\left(\mathrm{SpO}_{2}\right)<0.92-0.94$ and showed no difference in duration of oxygen therapy nor length of hospital stay between HFNC and LFNP. The question remains whether HFNC is effective in severe respiratory distress or could be used as rescue treatment to prevent the need for invasive ventilation.

No consensus guidelines are available on procedures for starting, dosing or weaning of HFNC. Many local protocols recommend to refer to a PICU when the respiratory distress does not improve within 2 hours after initiation of HFNC $(10,11)$. Whether this timing is optimal or may be extended is unclear in the absence of relevant follow-up data.

Apart from patients with bronchiolitis, we also used HFNC in children with pneumonia and asthma. We hypothesized that HFNC as a rescue treatment can be safely administered at a pediatric ward of a general hospital.

\section{Methods}

We performed a retrospective chart review, including all patients $<18$ years who received HFNC between January 2015 and May 2016. We collected data on demographic and clinical characteristics, history of prematurity, relevant comorbidities, pediatric early warning score (PEWS) $(12,13)$ and outcome concerning PICU admissions and length of hospital stay.

\section{Setting:}

The pediatric department of Isala has 54 beds, with facilities for continuous monitoring of vital functions, with approximately 3800 pediatric admissions per year.. Although Isala has a neonatal and adult intensive care, it has no PICU. In the Netherlands, PICUs are only available in university hospitals, which are all at least $100 \mathrm{~km}$ away from Isala. We refer 15 to 25 patients each year to the PICU. The use of dry gas through low flow nasal prongs (LFNP), with a maximum flow rate of $2-3 \mathrm{~L} / \mathrm{min}$, is our standard method of oxygen administration. HFNC wasis started in case of severe respiratory distress, with impending respiratory failure, as assessed by the attending pediatric physician after admission to the pediatric ward. Patients with respiratory failure at the emergency department are transferred to the ward 
or ICU, immediately when oxygen administration through LFNP or non-rebreathing mask is insufficient. When HFNC fails, invasive mechanical ventilation for children can be initiated at Isala's intensive care for adults, but when duration of mechanical ventilation is expected to take more than 24 hours, patients are transported to a PICU.

\section{High flow}

In January 2015, the use of HFNC was implemented at our pediatric ward. HFNC is applied in children with severe respiratory distress due to bronchiolitis, pneumonia, or asthma. We used Optiflow in the first season (January-April 2015) and switched to Airvo (both from Fisher and Paykel, Auckland, New Zealand) in the second (from October 2015-May 2016). Apart from a small difference in the minimum flow rate (Airvo $2 \mathrm{~L} / \mathrm{min}$, Optiflow $0.5 \mathrm{~L} / \mathrm{min}$ ), both devices are comparable. All nurses at the pediatric ward were instructed how to use the devices by a pediatrician. High flow was started with $100 \%$ oxygen at a flow rate calculated per $\mathrm{kg}$ bodyweight: $2 \mathrm{~L} / \mathrm{kg} /$ minute for the first $10 \mathrm{~kg}$; and $0.5 \mathrm{~L} / \mathrm{kg}$ for every $\mathrm{kg}$ bodyweight above $10 \mathrm{~kg}$, with a maximum of 50L/min, according to the guideline of the PICU at the nearest by tertiary pediatric Hospital. After the patient was stabilized, the first step in weaning was to decrease the fraction of inspired oxygen $\left(\mathrm{FiO}_{2}\right)$ from $100 \%$ to $40-60 \%$, guided by oxygen saturations $(>94 \%)$, while maintaining the flow rate. Inability to wean $\mathrm{FiO}_{2}$ was a reason to consider referral to the PICU. In the first season, we gradually decreased flow with 0.5-1.0 liters per hour when work of breathing and respiratory rate improved, until a flow rate of $2 \mathrm{~L} / \mathrm{min}$; after which we could switch to LFNP $2 \mathrm{~L} / \mathrm{min}$ and further wean the flow until supplemental oxygen was no longer needed. In the second season, we didn't gradually decrease the flow but decreased it instantaneously to a flow rate of $2 \mathrm{~L} / \mathrm{min}$ with $\mathrm{FiO}_{2}$ $100 \%$. If work of breathing worsened, we increased the flow rate to the previous setting. When the child was stable with $\mathrm{FiO}_{2} 100 \%$ and flow rate $2 \mathrm{~L} / \mathrm{min}$, we switched to LFNP to further wean the flow until supplemental oxygen was no longer needed.

\section{PEWS}

According to our local protocol-in agreement with the regional PICU- the response of HFNC was evaluated 2 hours after the start to consider to either continue HFNC or to refer the patient to a PICU. The pediatric nurse assessed the patients' vital functions by documenting a Pediatric Early Warning Score (PEWS). The PEWS is a clinical scoring system (scale 0-28) based on work of breathing and vital functions such as heart rate, respiratory rate, oxygen saturation, need for oxygen, capillary refill time, blood pressure and temperature $(12,13)$. Nurses are instructed to consult the pediatrician immediately when the PEWS is 8 points or higher, and to assess the PEWS hourly when it is 6 points or higher. Although this PEWS was developed and validated for use as an early warning score and not for monitoring effect of high flow, we hypothesized it would give comprehensive information about clinical deterioration or improvement. Based on experience and consensus we defined a 2-point decrease in PEWS to be of clinical relevance. To assess safety of HFNC we documented any adverse events related to HFNC. We considered the following adverse effects to be related to use of HFNC: pneumothorax, abdominal distension and mucosal injury with nasal bleeding and ulceration (6). 
Therapy failure was defined as a referral to the PICU. Due to non-normal distribution we used Pearson's Chi-square test or the Fisher's exact test as appropriate to compare categorical data and we used the Mann-Whitney U-test for continuous data. We applied logistic regression to determine predictive factors of therapy failure. Statistical Package for the Social Sciences (SPSS) version 22 was used for statistical analyses.

\section{Results}

\section{Study population}

During the 16-month study period we used HFNC in 39 patients $(64.1 \%$ male, median age 6.3 months [interquartile range, IQR 3-20.6]). Almost halfSeventeen (46.2\%) of patients (42\%) had either a history of prematurity ( $<37$ weeks of gestation, $n=4$, of whom one with chronic lung disease), or other relevant comorbidity such as syndromal disease (Down $n=3$, Angelman $n=1$, Peter Plus $n=1$, unknown etiology $n=4$ ), chronic lung neuromuscular $(n=1)$ or congenital heart disease (coarctatio aortae $n=2$, vascular ring $n=1$ ). Two patients were admitted twice with HFNC, resulting in a total of 41 hospital admissions (table 1). All included patients had oxygen saturations $<92 \%$ upon admission. None of the patients had any adverse events related to HFNC. 18 patients (43.9\%) were referred to a PICU, after a median duration between HFNC initiation to PICU referral of 12.3 hours [IQR 3.8-33.4], and 16 of these PICU referrals received invasive mechanical ventilation.

One 4-week-old patient, not yet immunized, died from pertussis after 26 days of PICU admission.

\section{Two winter seasons}

There were $21 \mathrm{HFNC}$ treatments during the first season and 20 during the second. We found no significant differences between the two winter seasons in PICU referrals $(52.4 \%$ in the first season and $35 \%$ in the second, $p=0.262$ ) or in the median PEWS after 24 hours of HFNC (6 [IQR 4-7] in the first season and 5 [IQR 4-7] in the second, $p=0.349$ ). Although HFNC was started earlier in the second season (median hours from admission to start of HFNC 6 [IQR 4-31] hours versus 22 [3-40.5] hours, $p=$ 0.092), the median PEWS at start of HFNC was not significantly different: 9 [IQR 6.3-10] in the first season and 8 [IQR 7-9] in the second $(p=0.512)$.

\section{Clinical course}

Clinical improvement (PEWS decrease of $>2$ points) was noted in $60 \%$ of patients after initiation of HFNC. One patient was referred to the PICU within one hour after starting HFNC. Figure 1 shows the clinical course of included patients. Treatment failure occurred in 10/24 (41.7\%) of patients initially improving and in 7/16 (43.8\%) of patients not improving within 2 hours of HFNC $(p=0.896)$. Treatment failure (PICU admission) was not significantly related to patient characteristics (age or comorbidity), timing of initiation of HFNC, or to the PEWS at start of HFNC (table 2). 
Figure 2 shows that half of the children who were referred to PICU were intubated within 8 hours after HFNC initiation. There was no difference in time of referral to PICU in the children who improved after 2 hours and those who did not $(p=0.379)$. The median length of PICU admission in patients with early referral (within 8 hours after the start of HFNC, $n=9$ ) was 6 days [IQR 3.5-17] compared to 9 days in patient referred after 8 hours of HFNC [IQR $8-13, p=0.88]$.

\section{Discussion}

\section{Safety of HFNC}

In this study on HFNC use in 41 pediatric patients on a general pediatric ward without direct access to $\mathrm{PICU}$, we found no adverse events or complications related to the use of HFNC, suggesting it is safe to initiate HFNC and to monitor these patients with respiratory distress in this clinical setting. Although the small study size precludes the formulation of firm conclusions, our data extends previous reports on HFNC safety $(4,5,7,14-17)$ by showing safe initiation of HFNC even without direct access to a PICU.

\section{Prediction of treatment failure and timing of PICU referral}

We found no significant predictors for treatment failure. Early initiation of HFNC at lower disease severity was not associated with better outcome. An initial response to HFNC (i.e., a decrease in PEWS after 2 hours) or a PEWS $<6$ was not associated with treatment success. Most treatment failures occurred between 2 and 24 hours after initiation of HFNC. These findings emphasize the need for longer follow up of treatment effect, at least until 24 hours after the start of HFNC, in studies evaluating HFNC. It also means that patients who seem to stabilize after 2 hours of HFNC should remain closely monitored because the risk of deterioration continues after this first phase (4). One of the reasons to recommend early PICU referral is the concern of sudden exhaustion during longer term HFNC treatment without a clear treatment benefit. Therefore, we compared the duration of PICU admission of the children referred to the PICU early ( $<8$ hours) to those referred later ( $>8$ hours) and found a nonsignificant trend of longer PICU admission in children referred early (median 6 vs 9 days, $p=0.088$ ). Our study therefore did not find evidence to support early PICU referral after initiation of HFNC therapy. Future, larger, studies will have to examine the consequences of a delay in referral to a PICU caused by HFNC.

We found no clinical factors that predicted HFNC failure. Three earlier studies in different settings examined risk factors for HFNC failure (18-20). In accordance with our findings, none of these found an association between HFNC failure and age at admission or a history of prematurity. In two earlier studies, a high respiratory rate at admission was a risk factor for intubation $(18,19)$, while the third did not find this association (20). We did not measure respiratory rate in isolation but only as a component of the composite PEWS, which was not associated with risk of PICU referral in our study, neither at admission nor at start of HFNC. The differences between studies can probably be explained by the different clinical settings, different severity of bronchiolitis and the different population sizes. 
The main strengths of our study are its setting in a general pediatric ward in secondary care without direct access to a PICU, and the inclusion of not only patients with bronchiolitis but also those with pneumonia and asthma. Also, our study examines the effect of HFNC as rescue therapy. The most important limitations of our study are its small sample size, and its retrospective design and the lack of a historic control group, which limited our ability to collect clinical data in a standardized fashion. The use of a composite score such as the PEWS hampers comparison with previous studies in which individual clinical signs were evaluated as potential risk factors.

\section{Conclusions}

Although with caution due to the small study sample and the retrospective methodology without controls, Tthis study suggests that the use of HFNC in children with severe respiratory distress is safe and feasible on a general pediatric ward without a nearby PICU facility. The clinical response to HFNC therapy should be monitored for at least 24 hours, both in clinical settings as in future HFNC trials. Due to our small sample size of 41 cases, our findings need to be confirmed in further studies.

\section{List Of Abbreviations}

$\mathrm{FiO}_{2}=$ Fraction of Inspired Oxygen

HFNC = High flow nasal cannula therapy

$\mathrm{IQR}=$ Interquartile Range

LFNP = Low Flow Nasal Prongs

PEWS $=$ Pediatric Early Warning Score

PICU = Pediatric Intensive Care Unit

$\mathrm{SpO}_{2}=$ Peripheral Capillary Oxygen Saturation

SPSS $=$ Statistical Package for the Social Sciences

\section{Declarations}

\section{Ethics approval and consent to participate}

Not applicable, we performed a retrospective chart review to evaluate our own management. Data was not gathered for the sake of research; ethics approval was not needed according to the Dutch Central Committee on Research Involving Human Subjects (CCMO). On their website they state: 'Retrospective research/research with patient files does not fall under the scope of the WMO as the research subject is not physically involved in the research. The data being researched are also not being gathered for the 
sake of the research.' Source: http://www.ccmo.nl/en/file-research?526591f4-32c8-4bcb-82ee$7 f 21 c 35 f f 8 b a$

We uploaded a letter of exemption which was provided by our IRB (The Daily Board of the Medical Ethics Committee Isala Zwolle).

\section{Consent to publish}

Not applicable

\section{Availability of data and materials}

The datasets used and/or analysed during the current study are available from the corresponding author on reasonable request.

\section{Competing interests}

None of the authors have competing interests.

\section{Funding}

No financial support was received.

\section{Authors' Contributions}

LMPK: Data collection, data entry, data analysis, wrote first draft of paper, manuscript production; RR: Primary data collection and data entry; VJL, PLPB: helped writing the manuscript; JB: data analysis and helped writing the manuscript

\section{Acknowledgements}

None

\section{References}

1. Hutchings FA, Hilliard TN, Davis PJ, et al.: Heated humidified high-flow nasal cannula therapy in children. Arch Dis Child 2015; 100:571-575

2. Beggs S, Wong ZH, Kaul S, et al.: High-flow nasal cannula therapy for infants with bronchiolitis. Cochrane database Syst Rev 2014; 1:CD009609

3. Mayfield S, JaunceyCooke J, Hough JL, et al.: High-flow nasal cannula therapy for respiratory support in children. Cochrane Database Syst Rev 2014; 3:CD009850

4. Kepreotes E, Whitehead B, Attia J, et al.: High-flow warm humidified oxygen versus standard low-flow nasal cannula oxygen for moderate bronchiolitis (HFWHO RCT): an open, phase 4, randomised controlled trial. Lancet $2017 ; 6736: 1-10$ 
5. Franklin D, Babl FE, Schlapbach LJ, et al.: A Randomized Trial of High-Flow Oxygen Therapy in Infants with Bronchiolitis [Internet]. N Engl J Med 2018; 378:1121-1131Available from: http://www.nejm.org/doi/10.1056/NEJMoa1714855

6. Mikalsen IB, Davis P, Øymar K: High flow nasal cannula in children: a literature review. Scand J Trauma Resusc Emerg Med 2016; 24:93

7. Mayfield S, Bogossian F, O'Malley L, et al.: High-flow nasal cannula oxygen therapy for infants with bronchiolitis: Pilot study. J Paediatr Child Health 2014; 50:373-378

8. Jhung MA, Sunenshine RH, Noble-Wang J, et al.: A National Outbreak of Ralstonia mannitolilytica Associated With Use of a Contaminated Oxygen-Delivery Device Among Pediatric Patients. Pediatrics 2007; 119:1061-1068

9. Carrillo A, Gonzalez-Diaz G, Ferrer M, et al.: Non-invasive ventilation in community-acquired pneumonia and severe acute respiratory failure. Intensive Care Med 2012; 38:458-466

10. Duke T, Evans J: Paediatric Intensive Care Unit ( PICU) High Flow Nasal Prong HFNP oxygen guideline [Internet]. 2014; 1-5[cited 2017 Nov 15] Available from: https://www.rch.org.au/picu/about_us/High_Flow_Nasal_Prong_HFNP_oxygen_guideline/

11. Colin G, Catarina S, Laura A, et al.: Nottingham Children's Hospital High Flow Nasal Cannula Therapy [Internet]. 2017; Available from: https://www.nuh.nhs.uk/handlers/downloads.ashx?id = 68942

12. Parshuram CS, Bayliss A, Reimer J, et al.: Implementing the Bedside Paediatric Early Warning System in a community hospital: A prospective observational study. Paediatr Child Health 2011; 16:e18-22

13. Fuijkschot J, Vernhout B, Lemson J, et al.: Validation of a paediatric early warning score: First results and implications of usage. Eur J Pediatr 2015; 174:15-21

14. Bressan S, Balzani M, Krauss B, et al.: High-flow nasal cannula oxygen for bronchiolitis in a pediatric ward: a pilot study. Eur J Pediatr 2013; 172:1649-1656

15. Hilliard TN, Archer N, Laura $\mathrm{H}$, et al.: Pilot study of vapotherm oxygen delivery in moderately severe bronchiolitis. Arch Dis Child 2012; 97:182-3

16. Kallappa C, Hufton M, Millen G, et al.: Use of high flow nasal cannula oxygen (HFNCO) in infants with bronchiolitis on a paediatric ward: a 3-year experience. Arch Dis Child 2014; 99:790-1

17. Campaña MB, Ortiz JO, Muñoz CN, et al.: High flow therapy versus hypertonic saline in bronchiolitis: randomised controlled trial. 2013; 99:511-515

18. Abboud PA, Roth PJ, Skiles CL, et al.: Predictors of failure in infants with viral bronchiolitis treated with high-flow, high-humidity nasal cannula therapy*. Pediatr Crit Care Med 2012; 13:e343-9

19. Kelly GS, Simon HK, Sturm JJ: High-Flow Nasal Cannula Use in Children With Respiratory Distress in the Emergency Department. Pediatr Emerg Care 2013; 29:888-892

20. Betters KA, Gillespie SE, Miller J, et al.: High flow nasal cannula use outside of the ICU; factors associated with failure. Pediatr Pulmonol 2016; 52:806-812

\section{Tables}




\begin{tabular}{|l|l|}
\hline Table 1 Characteristics of hospital admissions (n=41) in children treated with HFNC \\
\hline Diagnosis & $29(70.7)$ \\
\hline Bronchiolitis, number (\%) & $10(24.4)$ \\
\hline Pneumonia, number (\%) & $2(4.9)$ \\
\hline Asthma, number(\%) & $7[5-9]$ \\
\hline Pediatric Early Warning Score (PEWS) & $20(48.8)$ \\
\hline At admission, median [IQR] & $8[6.5-9]$ \\
\hline PEWS 8 or higher at admission, number (\%) & $8.7[4.2-14.3]$ \\
\hline PEWS at PICU referral, median [IQR] & 41 \\
\hline Length of stay, median [IQR] & $18(43.9)$ \\
\hline $\mathrm{O}_{2}$-saturation < 92\% & $12.3[3.8-33.4]$ \\
\hline PICU referrals, number (\%) & Hours from start HFNC until PICU referral, median [IQR] \\
\hline $\begin{array}{l}\text { PICU = Pediatric Intensive Care Unit; HFNC = High Flow Nasal Cannula; PEWS = Pediatric } \\
\text { Early Warning Score: scale O-28; nurses are instructed to call the pediatrician immediately } \\
\text { when PEWS 8. }\end{array}$ \\
\hline
\end{tabular}

\begin{tabular}{|c|c|c|c|}
\hline Clinical variable & $\begin{array}{l}\text { Odds } \\
\text { ratio }\end{array}$ & $\begin{array}{l}\text { 95\% Confidence } \\
\text { interval }\end{array}$ & $\begin{array}{l}\text { p- } \\
\text { value }\end{array}$ \\
\hline Age in months & 1.00 & 0.99 to 1.10 & 0.88 \\
\hline Medical history (prematurity or comorbidity) & 0.49 & 0.14 to 1.72 & 0.26 \\
\hline PEWS at admission & 0.92 & 0.75 to 1.12 & 0.40 \\
\hline $\begin{array}{l}\text { Duration of admission before start of HFNC in } \\
\text { hours }\end{array}$ & 1.01 & 0.99 to 1.04 & 0.31 \\
\hline PEWS at start of HFNC & 1.03 & 0.82 to 1.30 & 0.80 \\
\hline Change in PEWS 2 hours after start of HFNC & 1.16 & 0.86 to 1.58 & 0.33 \\
\hline \multicolumn{4}{|c|}{$\begin{array}{l}\text { PICU = Pediatric Intensive Care Unit; HFNC = High Flow Nasal Cannula; PEWS = Pediatric } \\
\text { Early Warning Score: scale 0-28; nurses are instructed to call the pediatrician immediately } \\
\text { when PEWS 8. }\end{array}$} \\
\hline
\end{tabular}

\section{Figures}




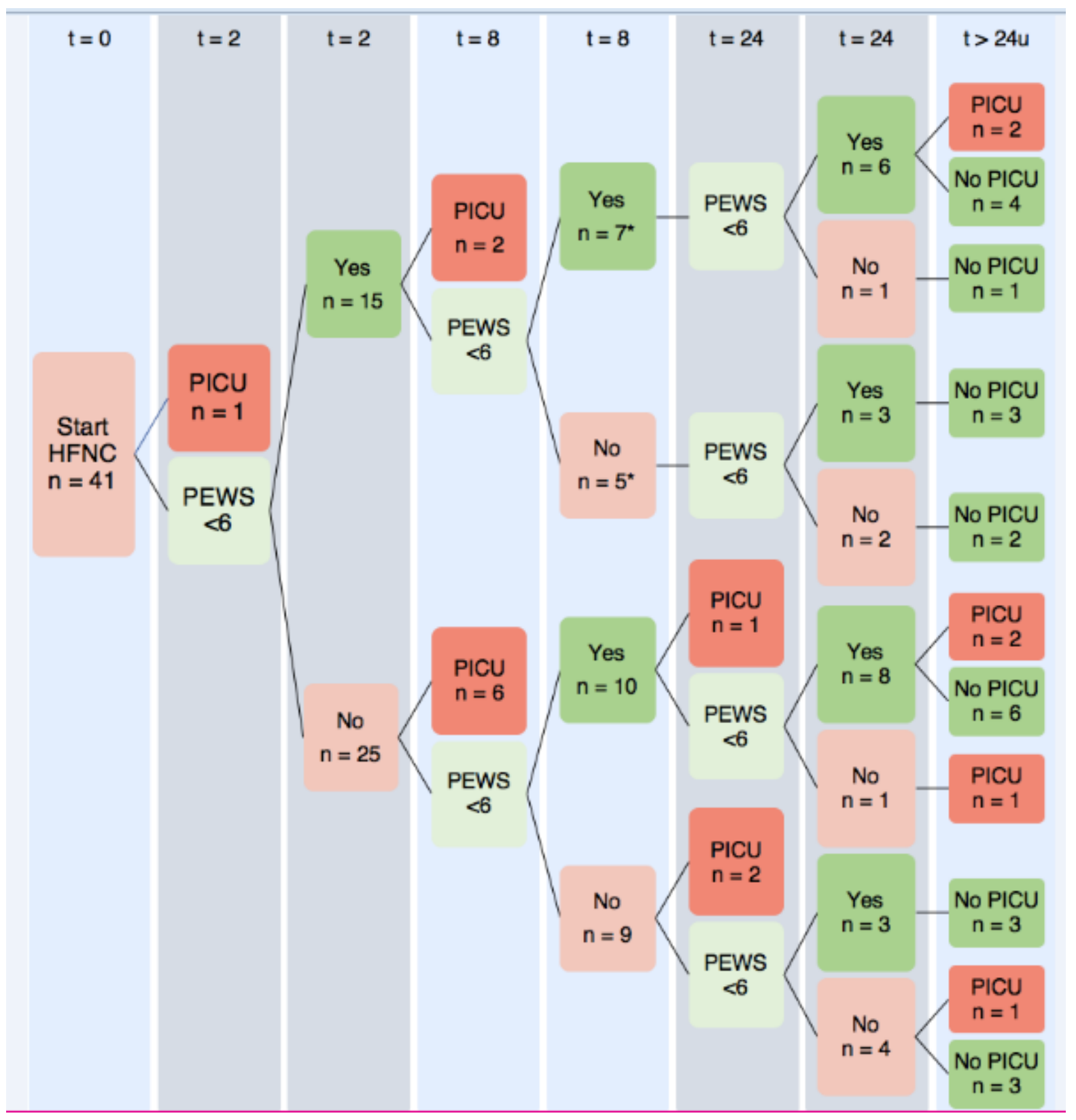

Figure 1

Clinical course of the included patients HFNC = High Flow Nasal Cannula, ' $t=0$ ' $=$ time of HFNC initiation; ' $\mathrm{t}=2$ ' = two hours after HFNC initiation, ' $\mathrm{t}=8$ ' = eight hours after HFNC initiation, ' $\mathrm{t}=24$ ' = 24 hours after HFNC initiation; ' $t>24$ ' = more than 24 hours after HFNC initiation. PICU = Pediatric intensive care unit PEWS = Pediatric Early Warning Score: scale 0-28; nurses are instructed to call the pediatrician immediately when PEWS $\geq 8$. * of one patient we missed data concerning $t=8$ and $t=24$ 


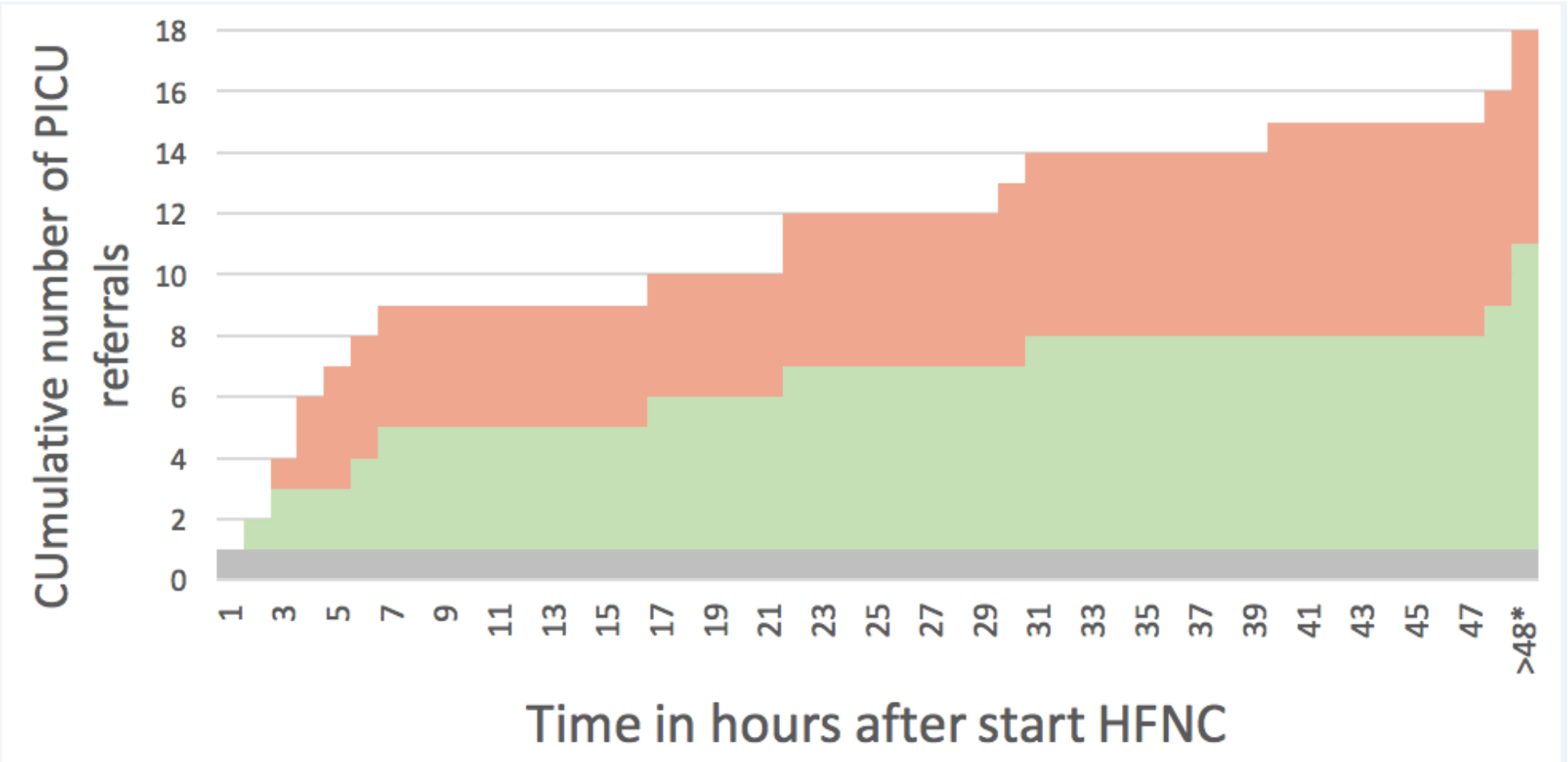

$\square * * \quad$ Improvement after 2 hours of HFNC $\quad$ No improvement after 2 hours of HFNC

\section{Figure 2}

Timing of referral to PICU after start of HFNC PICU = Pediatric Intensive Care Unit, HFNC = High Flow Nasal Cannula. Improvement was defined as decrease of at least 2 points in PEWS. ${ }^{\star}$ The17th patient was referred at 53 hours, and the 18th patient after 120 hours. ** One patient was referred after 1 hour of HFNC 